\title{
Parallelized iterative domain decomposition boundary element method for thermoelasticity
}

\author{
B. Gámez ${ }^{1,4}$, D. Ojeda ${ }^{1,4}$, E. Divo ${ }^{2}$, A. Kassab ${ }^{3}$ \& M. Cerrolaza ${ }^{4}$ \\ ${ }^{1}$ Departamento de Diseno Mecánico y Automatización, \\ Universidad de Carabobo, Venezuela \\ ${ }^{2}$ Department of Engineering Technology, \\ University of Central Florida, USA \\ ${ }^{3}$ Department of Mechanical, Materials, and Aerospace Engineering, \\ University of Central Florida, USA \\ ${ }_{4}^{4}$ National Institute of Bioengineering, \\ Central University of Venezuela, Venezuela
}

\begin{abstract}
The boundary element method (BEM) requires only a surface mesh to solve thermoelasticity problems, however, the resulting matrix is fully populated and non-diagonally dominant. This poses serious challenges for large-scale problems due to storage requirements and the solution of large sets of nonsymmetric systems of equations. In this article, an effective and efficient domain decomposition, or artificial sub-sectioning technique, along with a region-byregion iteration algorithm particularly tailored for parallel computation to address these issues are developed. The domain decomposition approach effectively reduces the condition number of the resulting algebraic systems, while increasing efficiency of the solution process and decreasing memory requirements. The iterative process converges very efficiently while offering substantial savings in memory. The iterative domain decomposition technique is ideally suited for parallel computation. Results demonstrate the validity of the approach by providing solutions that compare closely to single-region BEM solutions and benchmark analytical solutions.
\end{abstract}

Keywords: domain decomposition, thermoelasticity, parallel computation, boundary element method. 


\section{Introduction}

The boundary element method (BEM) is ideally suited to solve problems involving thermoelastic effects as the coupled problem can formally be solved entirely using a boundary discretization. This type of problem is encountered when a solid is subjected to heating conditions that give rise to a temperature distribution throughout its volume. This temperature distribution produces thermal expansions in the object under consideration. In an isotropic material, at a uniform reference temperature, $\mathrm{T}_{\text {ref }}$, a small uniform increase in temperature can produce a pure volumetric expansion if the object is not constrained against such movement. This expansion can be expressed as a so-called thermal strain $\left(e_{i j}^{T}\right)$, according to the equation:

$$
e_{i j}^{T}=\delta_{i j} \alpha \Delta=\delta_{i j} \alpha\left(T-T_{r e f}\right)
$$

where $\alpha$ is the thermal expansion coefficient. The expression above reveals that this thermal expansion can occur with absolutely no stresses present in the solid, Kane [1], Díaz et al [2]. In the standard BEM, the coefficient matrix is full and practical issues of storage and computation arise in large-scale modeling, particularly in 3D. Domain decomposition with explicit solution of the banded coefficient matrix and multipole methods have been used to successfully mitigate these problems. In this article, we propose an effective and efficient domain decomposition, or artificial sub-sectioning technique, along with a region-byregion iteration algorithm particularly tailored for parallel computation. The domain decomposition effectively reduces the condition numbers of the resulting algebraic systems, while increasing solution process efficiency and decreasing memory requirements. The iterative process converges very efficiently while offering substantial savings in memory and much promise for efficient solution of $3 \mathrm{D}$ thermoelasticity problems using the BEM and it is ideally suited for parallel computation.

\section{BEM in thermoelasticity}

The BEM can be utilized to resolve tractions, displacements and stresses on the boundary $\Gamma$ and in the internal points of a domain $\Omega$, Brebbia and Domínguez [3], based on a displacement boundary integral formulation for thermoelasticity. The thermoelastic problem is governed by the equilibrium equation:

$$
\frac{\partial \sigma_{i j}}{\partial x_{j}}=-b_{i}
$$

and the Hook's constitutive relation:

$$
\sigma_{i j}=\frac{2 \mu \nu}{(1-\nu)} \delta_{i j} e_{i i}+\mu e_{i j}
$$

where $\sigma_{i j}$ is the stress tensor, $b_{i}$ is the body force vector, $e_{i j}=\frac{1}{2}\left(\frac{\partial u_{i}}{\partial x_{j}}+\frac{\partial u_{j}}{\partial x_{i}}\right)$ is the strain tensor, $u_{i}$ is the displacement vector, $\delta_{i j}$ is the Kronecker delta, $\mu$ 
is the shear modulus, and $\nu$ is the Poisson ratio. Combining Eqs. (2) and (3) and introducing the strain tensor in terms of displacements yields the Navier's equation as:

$$
\mu \frac{\partial u_{i}}{\partial x_{j} \partial x_{j}}+\frac{\mu}{(1-2 \nu)} \frac{\partial u_{j}}{\partial x_{i} \partial x_{j}}=-b_{i}
$$

On each part of the boundary, $\Gamma$, either the displacement $u_{i}=\bar{u}_{i}$ on boundary $\Gamma_{u}$ or the traction $t_{i}=\bar{t}_{i}$ on boundary $\Gamma_{t}$, is prescribed in a well-posed problem. So that, $\Gamma=\Gamma_{t} \cup \Gamma_{u}$ is the boundary of the domain $\Omega$. Using the Somigilana identity, an integral relation between the displacements $u_{i}^{p}$ in a collocation point " $p$ " and the displacements $u_{i}$ and the tractions $t_{i}$ on all boundary $\Gamma$ is readily obtained with the body forces $b_{i}$ appearing formally as a domain integral:

$$
c_{i j}^{p} u_{i}^{p}+\oint_{\Gamma} H_{i j} u_{i} d \Gamma=\oint_{\Gamma} G_{i j} t_{i} d \Gamma+\int_{\Omega} G_{i j} b_{i} d \Omega
$$

where $G_{i j}$ and $H_{i j}$ are fundamental solutions in terms of displacement and traction respectively, and $t_{i}=\sigma_{i j} n_{j}$ is the traction vector and $n_{j}$ is the boundary outward normal vector; see Brebbia and Domínguez [3] and Cheng et al [4]. For thermoelasticity, the field stresses is:

$$
\sigma_{i j}=\sigma_{i j}^{e}+\sigma_{i j}^{T}
$$

where the first term $\sigma_{i j}^{e}$ represents the contribution to the stress components due to the actual straining of the material, while the last term $\sigma_{i j}^{T}$ represents the thermal expansion effect, Kane [1], which is given by:

$$
\sigma_{i j}^{T}=-m \delta_{i j}\left(T-T_{r e f}\right)
$$

Therefore the body forces in the Navier's equation will have the form:

$$
b_{i}=-m \frac{\partial T}{\partial x_{i}}
$$

where $m$ is the thermoelastic constant of the form:

$$
m=\frac{2 \mu \alpha(1+\nu)}{(1-2 \nu)}
$$

The domain integral can be expanded using Green's second identity and other transformations to finally obtain the boundary integral equation for displacements as:

$$
c_{i j}^{p} u_{i}^{p}+\oint_{\Gamma} H_{i j} u_{i} d \Gamma=\oint_{\Gamma} G_{i j} t_{i} d \Gamma+\frac{m}{k}\left[\oint_{\Gamma} E_{j} q d \Gamma-\oint_{\Gamma} F_{j}\left(T-T_{r e f}\right) d \Gamma\right]
$$

where $k$ is the material thermal conductivity. Moreover, assuming thermal equilibrium, $\partial^{2} T / \partial x_{i} \partial x_{i}=0$, the temperature a the collocation point $p$ can 
also be related to the temperature and heat flux at the boundary by means of the following boundary integral equation:

$$
c^{p} T^{p}+\oint_{\Gamma} G_{T} q d \Gamma=\oint_{\Gamma} H_{T} T d \Gamma
$$

where $c^{p}$ is a geometrical constant that possesses similar properties as $c_{i j}^{p}$, and $G_{T}$ and $H_{T}$ are the fundamental solutions for temperature and heat flux. The normal heat flux is defined as: $q=-k\left(\partial T / \partial x_{i}\right) n_{i}$. Additionally, one can obtain a BIE that relates the stresses to boundary displacements, tractions, temperatures and heat fluxes as:

$$
\begin{aligned}
c_{i j}^{p} \sigma_{i k}^{p}+\oint_{\Gamma} S_{i j k} u_{i} d \Gamma & =\oint_{\Gamma} D_{i j k} t_{i} d \Gamma \\
& +\frac{m}{k}\left[\oint_{\Gamma} A_{j k} q d \Gamma-\oint_{\Gamma} B_{j k}\left(T-T_{r e f}\right) d \Gamma\right] \\
& -c_{j k}^{p} m\left(T-T_{r e f}\right)^{p}
\end{aligned}
$$

where $S_{i j k}$ and $D_{i j k}$ are the fundamental solutions of stresses. The coefficients $E_{j}$, $F_{j}, A_{j k}$ and $B_{j k}$ can be derived directly of the fundamental solution $G_{i j}$, Brebbia et al[3]. The discretized displacement BIE can be formulated as:

$$
\begin{aligned}
c_{i j}^{p} u_{i}^{p} & =\sum_{l=1}^{N E} \sum_{n=1}^{N N} t_{i}^{l, n} \int_{-1}^{1} G_{i j}^{l}(\eta) M^{n}(\eta) J^{l}(\eta) d \eta \\
& -\sum_{l=1}^{N E} \sum_{n=1}^{N N} u_{i}^{l, n} \int_{-1}^{1} H_{i j}^{l}(\eta) M^{n}(\eta) J^{l}(\eta) d \eta \\
& +\frac{m}{k} \sum_{l=1}^{N E} \sum_{n=1}^{N N} q^{l, n} \int_{-1}^{1} E_{j}^{l}(\eta) M^{n}(\eta) J^{l}(\eta) d \eta \\
& -\frac{m}{k} \sum_{l=1}^{N E} \sum_{n=1}^{N N}\left(T^{l, n}-T_{r e f}\right) \int_{-1}^{1} F_{j}^{l}(\eta) M^{n}(\eta) J^{l}(\eta) d \eta
\end{aligned}
$$

where $N E$ is the number of elements and $N N$ is the number of degrees of freedom of the field variables in each element. For all examples presented in this paper, we utilize discontinuous quadratic elements with three $(N N=3)$ independent nodes for the field variables in each element with $M^{n}(\eta)$ denoting the respective quadratic shape functions. Also, $\eta$ denotes the homogeneous parametrization variable(s) of the element geometry, and $J^{l}(\eta)$ is the Jacobian of the element $\Delta \Gamma_{l}$. To form an algebraic system, the point $p$ is located at each of the $N N$ nodes of all the elements $N E$. This generates independent equations of the form:

$$
[H]\{u\}=[G]\{t\}+\{s\}
$$

The matrices $[H]$ and $[G]$ have dimensions $N \times N$ where $N=d \times N E \times N N$ and $d$ is the number of spatial dimensions ( 2 or 3 ). The vector $\{s\}$ contains 


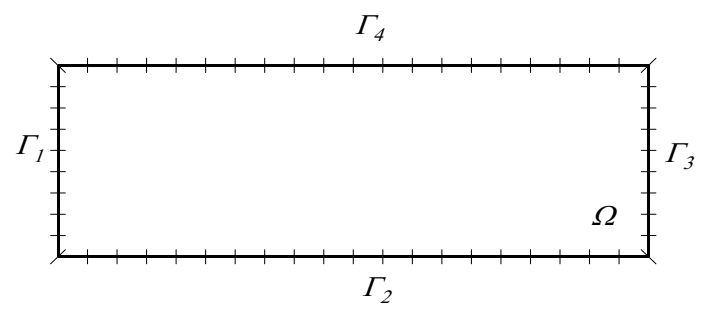

Figure 1: BEM single region discretization.

the integrated information of the thermal effects on the elastic field. Finally, the boundary conditions $\bar{t}_{i}$ or $\bar{u}_{i}$ are introduced in this algebraic system to arrive at the standard from: $[A]\{x\}=\{b\}$ where the unknown vector $\{x\}$ contains the strains and tractions that they were not specified as boundary conditions. Once the system is solved, all the field variables at the boundary are known and can be employed to determine strains and internal stresses using the appropriate boundary integral equations, Eqs. (10) and (12). It is important to emphasize that the same procedure is initially used to determine the temperature and heat fields using the temperature boundary integral equation.

\section{Iterative domain decomposition (IDD)}

To determine the IDD efficiency it is necessary first to indicate the requirements of memory for the resolution of the domain problem $\Omega$ in a single region, see Fig. 1, with the corresponding boundary conditions and the characteristic discretization of the boundary element method.

If a discretization of $N E$ elements with $N N$ independent nodes per each element is generated in a single region, the resulting algebraic system has dimensions $N \times N$, where $N=d \times N E \times N N$ and $d$ is the number of spatial dimensions ( 2 or 3$)$. So:

$$
\Omega \Rightarrow[A]_{N x N}\{x\}_{N x 1}=\{b\}_{N x 1}
$$

where the vector $\{x\}$ represents the unknown values of the tractions and displacements around the boundary. In this case the number of floating point operations required to arrive at the algebraic system above is proportional to $N^{2}$ as well as direct memory allocation also proportional to $N^{2}$. The solution of the algebraic system can be performed using a direct solution method such as LU decomposition requiring floating point operations proportional to $N^{3}$ or an indirect method such as Bi-conjugate Gradient or Generalized Minimum Residual (GMRes), which, in general, requires floating point operations proportional to $N^{2}$ to achieve convergence.

On the other hand, if a multi-region solution process is adopted instead, the original domain $\Omega$ is divided into $\mathrm{K}$ sub-domains $\Omega_{l} \therefore l=1 \ldots K$ separated by interfaces artificially created, and each one is independently discretized, as shown 


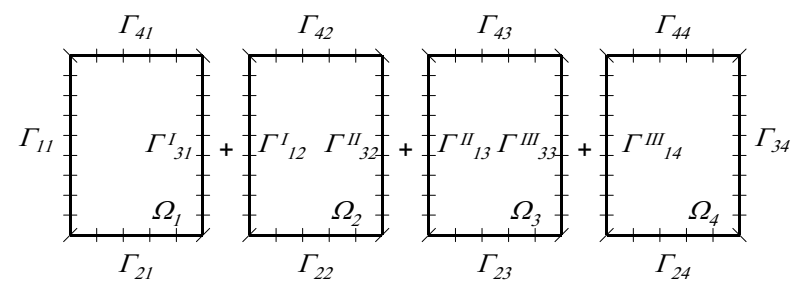

Figure 2: BEM domain decomposition and discretization.

in Fig. 2 for the case where $K=4$. Successively, the solution in each sub-domain can be obtained independently through a standard process, as long as the boundary conditions in the artificial interfaces between the sub-domain are imposed.

For example, the first sub-domain $\Omega_{1}$ in figure 2 is independently analyzed. The application of the boundary element method in this sub-domain is used to generate an algebraic system as follows:

$$
[A]_{n x n}\{x\}_{n x 1}=\{b\}_{n x 1}
$$

where the dimension $n=d \times n e \times N N$ is obviously a fraction of the original dimension $N$, Divo et al [7] provides a thorough explanation.

Naturally, the boundary conditions at the artificial interfaces between the subdomain are originally unknown, and, therefore a scheme must be devised to guarantee the continuity and equilibrium of the field variables between the subdomain, namely that:

$$
\begin{gathered}
u_{i}^{a}=u_{i}^{b} \\
t_{i}^{a}=-t_{i}^{b}
\end{gathered}
$$

where the superscripts $a$ and $b$ indicate each side of the interface at issue. In order to guarantee these conditions at each iteration including at the initial guess, a preliminary discretization of very low resolution is carried out providing a simplified model for the problem. This is solved by BEM to generate physically meaningful initial values at the interfaces. The latter are updated utilizing a refined discretization until a solution is achieved that satisfies the interfacial equilibrium and continuity conditions within a set tolerance.

The progression of the iterative process involves two stages. In the first stage, each interface is individually imposed with conditions of first kind prescribing displacements $\bar{u}_{i}$ and the tractions $t_{i}$ are solved using the standard boundary element method in each sub-domain. These intermediate computed tractions do not agree on each side of the interfaces, thus it is necessary to alter these tractions to force them to satisfy the equilibrium conditions, and this is accomplished using 
the following:

$$
\begin{aligned}
& \bar{t}_{i}^{a}=t_{i}^{a}-\frac{t_{i}^{a}+t_{i}^{b}}{2} \\
& \bar{t}_{i}^{b}=t_{i}^{b}-\frac{t_{i}^{a}+t_{i}^{b}}{2}
\end{aligned}
$$

This guarantees that the updated tractions, $\bar{t}_{i}^{a}$ and $\bar{t}_{i}^{b}$, have the same magnitude but opposite signs satisfying the equilibrium condition. Once these tractions are updated, the second stage of the iterative procedure utilizes these tractions as the imposed interfaces conditions for each sub-domain. A new displacement field in the interfaces is obtained, and, again, the displacements do not agree on both sides of each interface. They are updated by a simple average to ensure continuous displacement, so that:

$$
\begin{aligned}
& \bar{u}_{i}^{a}=\frac{u_{i}^{a}+u_{i}^{b}}{2} \\
& \bar{u}_{i}^{b}=\frac{u_{i}^{a}+u_{i}^{b}}{2}
\end{aligned}
$$

The iterative norm is defined as the root mean square measure between the updated displacements and the previous ones provides is defined by:

$$
L_{2}=\sqrt{\frac{1}{N N I} \sum_{l=1}^{N N I}\left(\bar{u}_{i}-u_{i}\right)^{2}}
$$

where $N N I$ is the number of nodes in the interfaces. The iteration is stopped when $\epsilon$ reaches a preset value.

If a direct approach such as LU factorization is employed for all sub-domains, the LU factors of the coefficient matrices for all sub-domains can be computed only once at the first iteration and stored on disk or in RAM for later use during the iteration process. Subsequently, only a forward and a backward substitution will be required. This feature provides a significant reduction in the computational burden for the overall BEM solution.

\section{Parallel implementation}

The domain decomposition BEM formulation detailed above is ideally suited to parallel computing, Erhart et al [5]. The algorithm has been coded using MPICH and implemented on a multiprocessor cluster comprised of dual 64 bit Xeon 3.2 $\mathrm{GHz}$ nodes equipped with $6 \mathrm{~GB}$ RDRAM running under the Fedora core 5 operating system. A static load-balancing routine is utilized to optimally distribute the computation over the nodes. This optimization is performed using a discrete Genetic Algorithm as described in Divo and Kassab [6]. A key step in the domain decomposition is to keep each sub-domain discretization to a number of elements that allows the problem to be stored in available RAM memory to avoid disk paging, Divo et al [7]. 


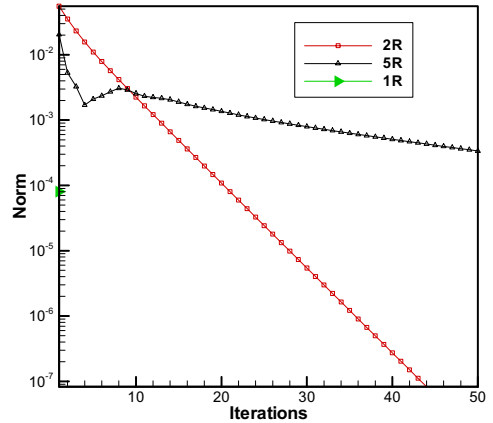

Figure 3: Elastic field convergence.

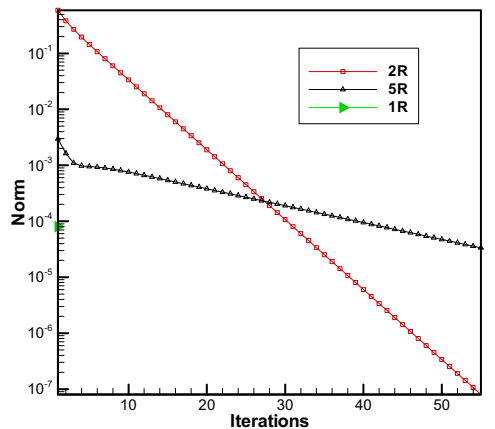

Figure 4: Thermal field convergence.

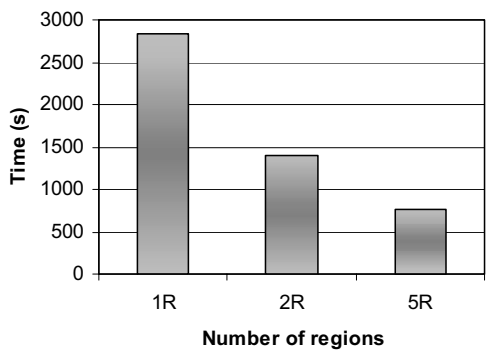

Figure 5: Time vs. number of regions.

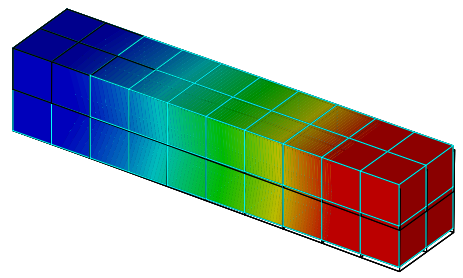

(a)

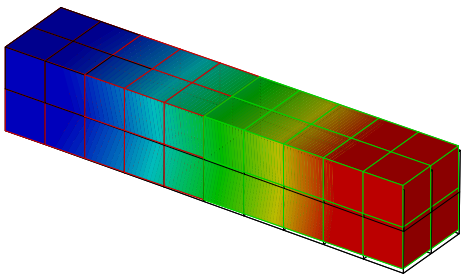

(b)

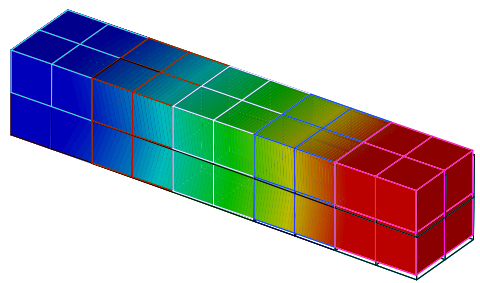

(c)

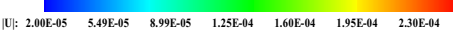

Figure 6: Contour plots of the displacement for: (a) oneregion, (b) two-region, (c) five-region cases.

\section{Numerical validation and examples}

The example presented in this paper, consists of a square cross-section cantilever beam whose height and width are $h=w=0.5 \mathrm{~m}$ and length $L=0.25 \mathrm{~m}$. This example is provided to verify the convergence and accuracy of the domain 
decomposition approach in 3-D. The beam is discretized with 88 biquadratic isoparametric discontinuous elements for a total number of degrees of freedom for the single-region case of $N=2112(3 \times 88 \times 8)$. The thermal conductivity is taken as $k=14.9 \mathrm{~W} / \mathrm{mK}$ and the thermal expansion coefficient as $\alpha=13 \cdot 10^{-6} \mathrm{~K}^{-1}$. The shear modulus is set to $\mu=60 G P a$ and the Poisson ratio is set to $\nu=0.3$. The model is solved in a single region and it is also decomposed into 2 and 5 sub-domains in the longitudinal direction. The reference temperature is $T_{r e f}=$ $373 \mathrm{~K}$. The left longitudinal surface of the beam is clamped and imposed with a temperature $T=373 \mathrm{~K}$. The rest of the surfaces are thermally insulated except for the top perimetral surface which is imposed with a heat flux $q=-1000 \mathrm{~W} / \mathrm{m}^{2}$. An uniform load of $P=1 \mathrm{MPa}$ is imposed on the top perimetral surface.

Plots of the norm as a function of iterations for the elastic and thermal fields are shown in Fig. 3-4 respectively. The norm decays rapidly for both multi-region cases. The convergence criterion used was $\epsilon=10^{-7}$ and was reached in less than 50 iterations for the two-region case and in less than 100 iterations for the fiveregion case. Contour plots of the displacements of the cantilever beam are shown for the one-region, two-region, and five-region cases in Fig. 6 (a)-(c).

It should be noted, in Fig. 5, that the two-region model corresponds to a computational time reduction of $50.81 \%$ when compared to a single region cantilever beam, while the five-region case provides a $73.07 \%$ reduction. It is noted that the total time to solution reported here includes load balancing, generation of $H$ and $G$ matrices, resolution of elastic and thermal field, and iteration to convergence.

\section{Conclusions}

In this article, we propose an effective and efficient BEM iterative domain decomposition (BEM-IDD) algorithm to solve large-scale 3-D BEM thermoelastic problems. In order to tackle large problems, the original domain is decomposed into a number of sub-domains. Results indicate that the proposed BEM-IDD technique is well-suited for parallel computation, converging efficiently and offering substantial savings in memory and computational time over traditional BEM formulation.

\section{Acknowledgements}

The work undertaken in this project was carried out under the institutional and financial support provided by the University of Central Florida (USA), University of Carabobo (Venezuela), and FONACIT (Venezuela).

\section{References}

[1] Kane, J., Boundary Element Analysis in Engineering Continuum Mechanics, Prentice-Hall, New Jersey 1994, pp. 123, 378. 
[2] Díaz, F., Yates, J.R., and Patterson E.A., "Some improvements in the analysis of fatigue cracks using thermoelasticity," International Journal of Fatigue, Vol. 26, 2004, pp. 365-376.

[3] Brebbia, C.A. and Domínguez, J. J., Boundary Element: An Introductory Course, Computational Mechanics Publ. Boston co-publisher McGraw Hill, New York, 1989, pp. 134-250.

[4] Cheng, A.H., Chen, C.S., and Golberg, M.A., Rashed, Y.F., "BEM for thermoelasticity and elasticity with body force-a revisit," Engineering Analysis with Boundary Elements, Vol. 25, 2001, pp. 377-387.

[5] Erhart, K., Divo, E., and Kassab, A., "A parallel domain decomposition boundary element method approach for the solution of large scale transient heat conduction problems," Engineering Analysis with Boundary Elements, Vol. 30, 2006, pp. 553-563.

[6] Divo, E. and Kassab, A., "A meshless method for conjugate heat transfer problems," Engineering Analysis with Boundary Elements, Vol. 29, 2005, pp. 136-149.

[7] Divo, E., Kassab, A., and Rodríguez, F.: "Parallel domain decomposition approach for large-scale three-dimensional boundary-element models in linear and nonlinear heat conduction," Numerical Heat Transfer, Part B., Vol. 44, 2003, pp. 417-437. 